\title{
Selective Hypertext Induced Topic Search
}

\author{
Amit C. Awekar \\ NC State University \\ Raleigh, NC 27695, USA \\ acawekar@ncsu.edu
}

\author{
Pabitra Mitra \\ Indian Institute of Technology \\ Kharagpur, India - 721302 \\ pabitra@cse.iitkgp.ernet.in
}

\author{
Jaewoo Kang \\ NC State University \\ Raleigh, NC 27695, USA \\ kang@csc.ncsu.edu
}

\begin{abstract}
We address the problem of answering broad-topic queries on the World Wide Web. We present a link based analysis algorithm SelHITS, which is an improvement over Kleinberg's HITS [4] algorithm. We introduce the concept of virtual links to exploit the latent information in the hyperlinked environment. We propose a novel approach to calculate hub and authority values. We present a selective expansion method which avoids topic drift and provides results consistent with only one interpretation of the query even if the query is ambiguous. Initial experimental evaluation and user feedback show that our algorithm indeed distills the most important and relevant pages for broad-topic queries. We also infer that there exists a uniform notion of quality of search results within users.
\end{abstract}

\section{Categories and Subject Descriptors}

H.3.3 [Information Storage and Retrieval]: Information Search and Retrieval-information filtering

\section{Keywords}

Link analysis, Searching, Topic Distillation

\section{INTRODUCTION}

Searching information on the WWW is now a common practice. Traditional information retrieval techniques are not sufficient for searching information on the WWW. Kleinberg's HITS algorithm [4] is a well known algorithm for answering broad-topic queries in hyperlinked environment. It can be improved by selectively choosing a candidate set of pages for ranking and novel approach to ranking the pages.

\section{KLEINBERG'S HITS ALGORITHM}

HITS algorithm [4] associates two scores with each page. Authority which is a measure of authoritative information contained in the page and $H u b$ which is a measure of links to good authorities. Using some existing search system, HITS algorithm gets a set of relevant pages for user query. This is referred to as the root set (Refer Figure 1). Then all the pages from one link neighborhood of the root set are added to the root set. This is referred to as the base set. Consider a page $P_{i}$. Let $\operatorname{Par}_{i}$ be set of pages which have hyperlink to $P_{i}$ and are present in the base set. Let $C h i_{i}$ be set of Copyright is held by the author/owner(s).

$W W W 2006$, May 22-26, 2006, Edinburgh, UK. pages that $P_{i}$ has hyperlink to and are present in the base set. Let hub value for $P_{i}$ be denoted as $H_{i}$ and authority value as $A_{i}$. Then $A_{i}=\sum_{l \in \operatorname{Par}_{i}} H_{l}$ and $H_{i}=\sum_{l \in C h i_{i}} A_{l}$. If $E$ is the adjacency matrix for the base set, then authority vector $V_{a}$ is calculated as $V_{a}=E^{T} E V_{a}$ and hub vector $V_{h}$ as $V_{h}=E V_{a}$.

\section{SELHITS ALGORITHM}

SleHITS algorithm is briefly outlined in Figure 2.

Considering just page to page connectivity ignores other latent information in the WWW e.g. If multiple pages from same host are present in the root set then it indicates that there exists a community of pages on that host which is relevant to query topic. This context of location of pages is ignored by HITS algorithm. To exploit such latent information in the hyperlinked environment, we propose a novel approach to calculate hub and authority values alongwith the concept of virtual links. Many pages added to the root set in the expansion step of HITS algorithm are irrelevant or noisy. They substantially degrade the quality of results. We should be selective in expanding the root set. For ambiguous queries we should be able to distill pure topic i.e. giving results consistent with only one interpretation of the query.

Text analysis based improvement proposed by $[2,3]$ for HITS algorithm can be used in conjunction with our algorithm as well.

\subsection{Novel Approach to Ranking}

We consider two types of links for ranking. Actual links are those hyperlinks which are actually present between different pages. Virtual links are the pseudo links that we hypothetically insert. If a page $P_{i}$ has actual link to page $P_{j}$ in the root set then we insert pseudo links from $P_{i}$ to all other pages in the root set which reside on the same host as that of $P_{j}$. Considering virtual links helps exploit the context of location of a page.

For a given root set, consider adjacency matrix $E$. $E[i, j]=$ 1 if $P_{i}$ has actual link to $P_{j}$. We define modified adjacency matrix $Z$ such that $Z[i, j]=1$ if $P_{i}$ has actual or virtual link to $P_{j}$. Then hub and authority vectors for the root set are calculated as shown in Figure 3. Same can be represented in equation form as $V_{a}^{\prime}=Z^{T} Z V_{a}^{\prime}$. First calculating pseudo authority values assigns same authority value to all pages on one host as we are considering virtual links. Then actual hub values are calculated using these pseudo authority values as $V_{h}=E V_{a}^{\prime}$. This calculation of hub vector boosts hub values for those pages which have hyperlink to hosts which 


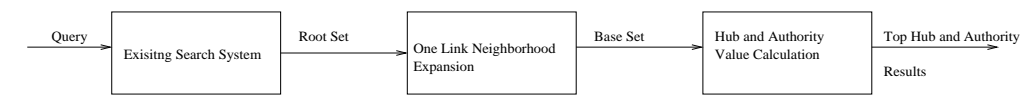

Figure 1: HITS Algorithm

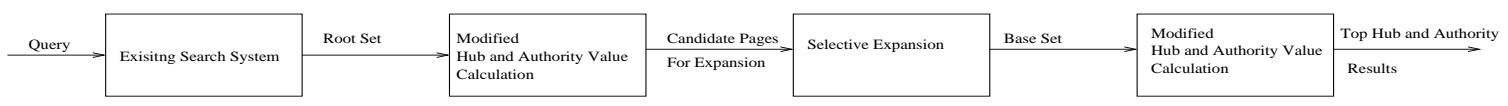

Figure 2: SelHITS Algorithm

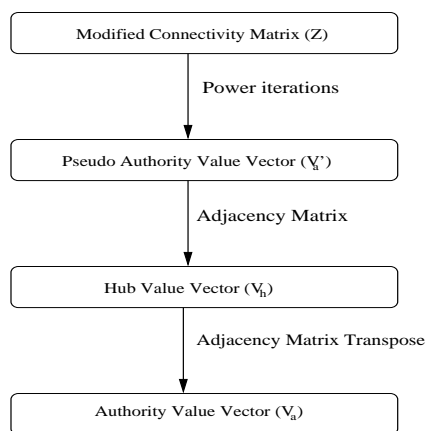

Figure 3: Calculating Hub and Authority Values

contain more pages in root set. Finally actual authority values are calculated as $V_{a}=E^{T} V_{h}$. This is required as initial authority values of some pages are unnecessarily boosted because of virtual links. We calculate hub and authority values twice, once on the root set and once on the base set.

\subsection{Selective Expansion}

After calculating hub and authority values on the root set, we select top 20 hubs and top 20 authorities for expansion. Good hubs point to good authorities [4], so we add outlinks of top 20 hubs to the root set. Good authorities are pointed by good hubs [4], so we add inlinks of top 20 authorities to the root set. As compared to HITS algorithm, this selective expansion procedure drastically reduces size of the base set and avoids topic drift as irrelevant pages are not added to the root set.

If query is ambiguous then we have corresponding disjoint communities in the link structure [4]. Top hubs and authorities are from same community as hub and authority values are mutually reinforcing. Selective expansion procedure adds new pages from single community, which further boosts hub and authority values for that community. Hence we are able to give results consistent with only one interpretation of the query. If user is interested in other interpretation of the query then we can simply remove current community from candidate pages and again run our algorithm.

\section{EXPERIMENTS AND CONCLUSION}

We tested our algorithm for 9 sample queries. We observed significant topic drift with HITS algorithm for these queries. For each query we generated top 20 hubs and top 20 authorities by SelHITS algorithm. Each query results were evaluated by 3 different users on the scale of 0 to 10 . Figure

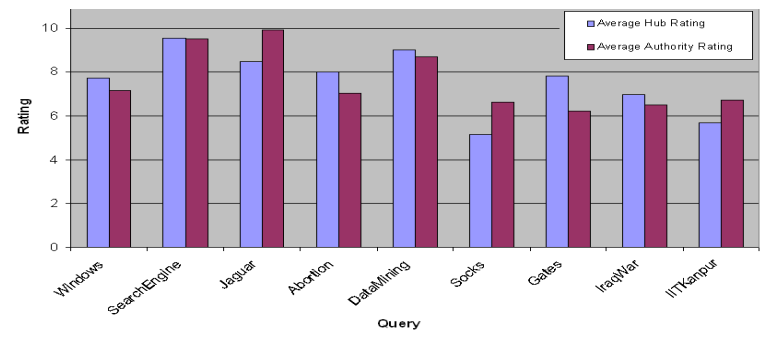

Figure 4: Average Ratings for Hub and Authorities

4 shows average scores for each query. As we can see within each query, scores for hubs and authority are similar. Also we noticed that scores of different users for a query were also similar. So we can infer that there exists a notion of quality of search results within users. For ambiguous queries we got results consistent with only one interpretation of the query. e.g. For query "gates" all results were related to "Bill Gates". More details about experimental results are available at [1].

We briefly discussed SelHITS algorithm which is an improvement over Kleinberg's HITS algorithm for answering broad-topic queries. Novel approach to calculate hub, authority values and selective expansion of the root set are the main features of this algorithm. Brief experimental evaluation suggests that algorithm performs satisfactorily. We are currently working on extensively testing the proposed algorithm for variety of queries and applying same algorithm for focused crawling problem.

\section{REFERENCES}

[1] More details about experiments on SelHITS algorithm. htttp://www4.ncsu.edu/ acawekar/SelHITSResults/.

[2] K. Bharat and M. R. Henzinger. Improved algorithms for topic distillation in a hyperlinked environment. In SIGIR '98: Proceedings of the 21st annual international ACM SIGIR conference on Research and development in information retrieval, pages 104-111, New York, NY, USA, 1998. ACM Press.

[3] S. Chakrabarti, B. Dom, P. Raghavan, S. Rajagopalan, D. Gibson, and J. Kleinberg. Automatic resource compilation by analyzing hyperlink structure and associated text. Comput. Netw. ISDN Syst., 30(1-7):65-74, 1998.

[4] J. M. Kleinberg. Authoritative sources in a hyperlinked environment. Journal of the ACM, 46(5-7):604-632, 1999. 